\title{
Two factors in the perception of velocity at a distance
}

\author{
WILLIAM EPSTEIN \\ University of Wisconsin, Madison, Wisconsin 53706
}

\begin{abstract}
Six experiments dealing with the effect of the surrounding framework on the perceived velocity of movement were reported. Experiment 1 reproduced Brown's (1931) transposition of velocity effect. Experiment 2 demonstrated the application of relational determination to the explanation of speed constancy. Experiment 3 showed that the relational effect is independent of the perceived sizes and perceived distances of the frameworks of movement. Only relative retinal size need be considered. Experiment 4 showed that relational factors can generate large deviations from speed constancy. Experiments 5 and 6 showed that only the immediately proximate frame needs to be taken into account in assessing effects on velocity. Despite the successes of the hypothesis of relational determination, it was argued that two factors are needed to explain perceived velocity: relative object-relative displacement accounting for perceived relative velocity, and a distance calibration mechanism explaining perceived absolute velocity.
\end{abstract}

Perceived velocity is not affected much by the distance between the observer and the plane in which the object is moving. Both laboratory evidence (Rock, Hill, \& Fineman, 1968) and everyday experience confirm this claim. My office window affords ready inspection of a typical city street extending for approximately $200 \mathrm{~m}$ from the window in my saggital plane. It is a common occurrence for persons to cross from one side of the street to the other, moving in a place which on the average is perpendicular to my line of sight. Since persons cross at different points, there is frequent opportunity to observe whether perceived differences in speed of walking are on the average associated with differences in the distances between the walker and myself. Despite the fact that the absolute angular velocity (the rate of retinal displacement) varies inversely as a function of viewing distance, I have never observed differences in walking velocity to vary systematically with viewing distance. Informal interrogation of other observers has yielded reports of similar experiences. Although some observers report that the walker's pace appears slower at the greater viewing distances, no one has ever reported a decrease of velocity which even roughly approximates the decrease of angular velocity associated with distance.

Figure 1 is a photograph of the scene from the window. Take this photograph to represent the frontoparallel projection of the scene for a monocular viewer. Considered in this way, the photograph suggests that a basis for perceived invariance of velocity may exist in optical stimulation. The photograph reveals that, just as angular velocity is inversely related

This work was supported by NIMH Research Grant 5RO1 MH 26703.

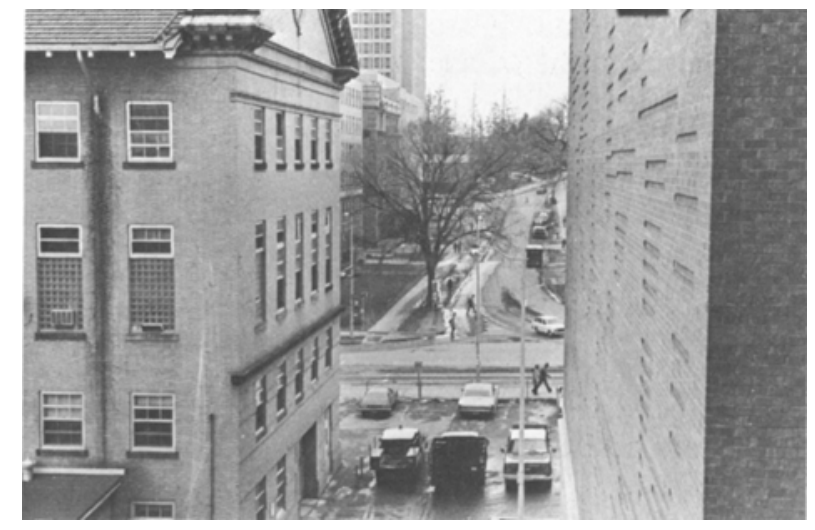

Figure 1. Photograph of Charter Street from fourth-floor window of Psychology Building. (University surveyors give the distance from the wall of the Psychology Building containing the office window to the second intersection as $183 \mathrm{~m}$. The height of the window from street level is $14 \mathrm{~m}$.)

to objective distance (D), so is the extent of the projection of the plane-of-crossing. Consequently, the proportion of the retinal field of movement traversed per unit of time will be the same for objects moving at the same linear velocity whatever their distance from the observer may be. This account of perceived velocity has been advocated explicitly by Wallach $(1939,1959)$ and is implied in a more general form by Gibson $(1950,1966)$. The principal support for Wallach's hypothesis of relational determination is Brown's (1931) demonstration of the dependence of perceived velocity on the relative size of the movement displays. Brown discovered that if all the linear dimensions of a movement field are transposed, e.g., doubled, then the velocity has to be transposed in approximately the same proportion in order to maintain equality of perceived velocity. As Wallach (1939) 
noted, if transposition is applied to retinal fields, speed constancy can be derived from the transposition principle.

The argument may be clarified by reference to Figure 2. In both arrangements pictured in Figure 2, the solid lines represent the objective dimensions of the frame, the dashed lines represent the retinal projections. In the transposition arrangement, the physical sizes of the frames are in a $2: 1(\mathrm{~L}: \mathrm{S})$ ratio while the distances are equal; in the constancy arrangement, the physical distances are in a $2: 1(\mathrm{~F}: \mathrm{N})$ ratio while the sizes are equal. The transposition effect shows that the two velocities appear equal only when the angular velocity in $L$ is twice that of $S$. Since the retinal situation in the constancy arrangement is identical to the transposition arrangement, the same result should prevail. Velocity in F should appear equal to velocity in $\mathrm{N}$ when the angular velocity in $F$ is one-half of the angular velocity in $N$. This is precisely the case when the objective linear velocities in $\mathbf{N}$ and $\mathbf{F}$ are equal. Thus, constancy is predicted from the transposition principle applied to retinal stimulation.

Although Wallach's $(1939,1959)$ account is frequently mentioned (e.g., Hochberg, 1978, pp. 120-123) in discussions of speed constancy, there have been no reports of direct examinations of Wallach's hypothesis and studies of Brown's transposition effect have been almost as rare. Cohen (1964) and Rock (1975, Chapter 5) have reviewed the literature. The principal aim of the series of experiments described here was to remedy these deficiencies.

Experiment 1 was designed to reproduce the transposition effect under conditions comparable to those in Brown's experiments.

Experiment 2 studied the effect of viewing distance and the effect of the availability of distance cues on the perception of velocity. In part, the conditions created in this experiment constituted an abstract version of the situation suggested by the photograph of the street scene.

Experiment 3 was conducted to show that neither the perceived size nor the perceived distance of the

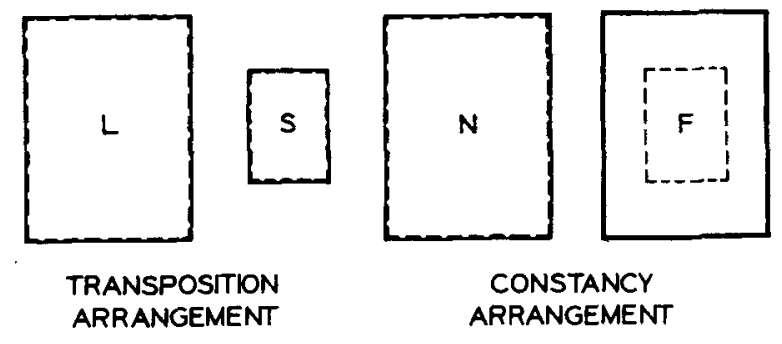

Figure 2. Comparison of distal and proximal dimensions in transposition demonstration and speed constancy demonstration. (Solid lines represent distal dimensions, dashed lines represent the retinal dimensions.) frame needs to be considered in deriving perceived velocity.

Experiment 4 was designed to demonstrate that the relational hypothesis can predict striking deviations from speed constancy.

Experiments 5 and 6 examined the effect on perceived velocity of contours outside the immediately proximate surroundings in a transposition arrangement and in a speed constancy arrangement.

\section{GENERAL PROCEDURE}

\section{Stimulj}

All experiments presented two displays for comparison. The moving component of each display was a circular point of light projected onto the back of a translucent screen by an optical system from the face of a Tektronix 604 monitor. The size of the spot could be varied by manipulating the focus of the optical system and the electronic focus of the CRT. The movement of the spot was controlled by a small on-line lab computer with a point-plot oscilloscope controller (PDP-11/10 with AA11k option).

Several different viewing conditions were used in this series of experiments, but they all share some common features. One display was assigned as the standard. This display was viewed at one of the following distances: $1,2,3,4$, or $5 \mathrm{~m}$. The display was always partially occluded by an opaque cardboard mask of one of the following dimensions: $7.5 \times 2.5,15 \times 5,22.5 \times 7.5$, $30 \times 10$, or $37.5 \times 12.5 \mathrm{~cm}$. The longer dimension was always oriented horizontally. The edges of this mask were painted with luminescent paint and were visible as luminous rectangles when the subject viewed the displays in the dark. The point of light on the standard display was $.85 \mathrm{~cm}$ in diameter $\left(.5^{\circ}\right.$ visual angle) for the smallest frame size, and proportionately larger for the larger frame sizes.

The second display was assigned as the comparison display. This display was viewed from a distance of $1 \mathrm{~m}$. Unless otherwise designated, the comparison display was a luminous frame surrounding a projected spot of light. The size of the comparison display is specified in the description preceding each experiment.

Two different viewing conditions could be imposed upon the subject. In the binocular viewing condition, the subject viewed the displays successively with both eyes and was free to make head movements. In the monocular condition, the subject viewed the displays successively through a single small aperture of approximately $.8 \mathrm{~cm}$ diam. The small size of the aperture required that the subject maintain an almost stationary head in order to keep the entire display in view.

\section{Procedure}

Each of the six experiments tested a different group of subjects. None of the subjects tested in a particular experiment had been exposed to similar experimental arrangements or procedures previously. With the exception of Experiment 3, which employed the method of magnitude estimation, the method of adjustment was used. The subject was instructed to adjust the velocity of the point of light moving across the comparison display to match the velocity of the point of light moving across the standard. In all cases, the point of light started at the right end of the mask, proceeded across the screen to the opposite end of the mask, and, after a short time interval, started another pass across the screen in the same direction. This movement was repeated on both screens, with the subject free to look at either of the screens as often as necessary. The subject adjusted the velocity of the comparison by adjusting a potentiometer. When the subject was satisfied that the velocities of the two displays matched, the trial was terminated by depressing a switch, which would clear the two displays and, after a period of $2.5 \mathrm{sec}$, start the next trial. The 
velocity determined by the potentiometer position was multiplied by a random number on each trial to assure that the position of the potentiometer could not be used as a reference for velocity between trials.

\section{EXPERIMENT 1}

\section{Method}

Stimuli. The standards were the five rectangular frames ranging from $7.5 \times 2.5$ to $37.5 \times 12.5 \mathrm{~cm}$ and presented individually at a distance of $1 \mathrm{~m}$ from the subject. Each frame enclosed a projected oscilloscope spot which traversed the entire frame at a linear velocity of 2.5 or $7.5 \mathrm{~cm} / \mathrm{sec}$. The frame in the variable display was the $7.5 \times 2.5 \mathrm{~cm}$ rectangle presented at a distance of $1 \mathrm{~m}$ from the subject and separated by $90^{\circ}$ from the standard display. The two displays were viewed successively and were the only visible objects in an otherwise dark room.

Subjects. The subjects were three men and three women undergraduate students.

Procedure. Each subject provided 40 judgments of velocity ( 5 standard frame sizes $\times 2$ standard velocities $\times 4$ replications) by adjusting the velocity in the variable display to match the velocity in the standard. Viewing was binocular and instructions stressed matching of phenomenal velocity. The subject was allowed as much time as he wished.

\section{Results}

The velocity settings of the variable were expressed as velocity ratios, i.e., $\mathrm{V}_{\mathrm{st}} / \mathrm{V}_{\mathrm{var}}$, and an analysis of variance was computed on these ratios. Only the ratio of frame sizes had a significant effect $[F(4,20)$ $=16.85, \mathrm{p}<.001]$. Since the velocity in the standard display did not affect the obtained velocity ratio, the averages for the two velocities at each frame size are shown in Table 1. When the frames were the same size, a velocity ratio of 1.007 was obtained indicating that in the absence of perturbing factors the task can be performed very accurately. Doubling the size of the standard display led to approximately a $45 \%$ increase of perceived velocity. The effects were substantial, although they fell short of Brown's results shown in the right-hand column of Table 1 and obviously deviated considerably from complete transposition of velocity. ${ }^{1}$

\section{Conclusion}

Transposition of the linear dimensions of the standard display had systematic effects on relative perceived velocity. The effects were in the direction predicted by Brown's (1931) transposition of velocity principle.

\section{EXPERIMENT 2}

The aims of Experiment 2 were to show that constancy of perceived velocity will be observed when a framed display of unvarying size is presented at varying egocentric distances and also to show that, for framed displays, constancy is not reduced by minimization of distance cues.

\section{Method}

Stimuli. The standard was the $37.5 \times 12.5 \mathrm{~cm}$ frame ptesented at a distance of $1,2,3,4$, or $5 \mathrm{~m}$ from the subject and enclosing a projected spot which traversed the frame at rates of 2.5 or $7.5 \mathrm{~cm} / \mathrm{sec}$. The variable display was the same physical size as the standard and was always located at a distance of $1 \mathrm{~m}$ from the subject and separated from the standard by $90^{\circ}$. The two displays were viewed successively in an otherwise dark room

Subjects. The subjects were 12 undergraduates, 6 assigned to a binocular viewing condition, 6 assigned to a monocular viewing condition.

Procedure. Each subject provided 40 judgments of velocity (5 standard distances $\times 2$ standard velocities $\times 4$ replications) by the method of adjustment. For half the subjects, viewing was binocular, for half of the subjects, viewing was monocular. Under the monocular condition, the subject viewed the displays through an aperture, $.8 \mathrm{~cm}$ in diameter, under instructions to avoid head movement during inspection of the display.

\section{Results}

Perfect constancy calls for standard:variable linear velocity ratios of 1 ; zero constancy calls for velocity ratios that match the ratios of the distances of the two displays. For example, when the standard display was presented at the distance of $5 \mathrm{~m}$ and the angular velocity is reduced in the same proportion, zero constancy would be manifest in a setting of the variable that was actually only one-fifth the linear velocity of the standard. Table 2 lists the perfect constancy and the zero constancy predictions and the velocity ratios obtained under the binocular and monocular conditions of viewing. The results were unequivocal: Constancy of velocity was nearly perfect for both viewing conditions.

\section{Conclusion}

Constancy of perceived velocity prevails when relative angular velocity remains constant even when information about egocentric (absolute) distance is reduced by restricting binocular input.

Table 1

Ratio of Velocities (Standard/Variable) for Perceived Equality of Velocity in Conventional Arrangement (Experiment 1)

\begin{tabular}{|c|c|c|c|c|c|}
\hline \multirow{2}{*}{$\begin{array}{l}\text { Ratio of Projective } \\
\text { Sizes of Frames } \\
\text { (Standard/Variable) }\end{array}$} & \multirow{2}{*}{$\begin{array}{c}\text { Transposition } \\
\text { Ratio }\end{array}$} & \multirow{2}{*}{$\begin{array}{l}\text { Linear } \\
\text { Velocity } \\
\text { Ratio }\end{array}$} & \multicolumn{2}{|c|}{ Obtained Ratio of Velocities } & \multirow{2}{*}{$\begin{array}{l}\text { Brown's } \\
\text { Results* }\end{array}$} \\
\hline & & & Mean & $\mathrm{SD}$ & \\
\hline 1 & 1 & 1 & 1.007 & .106 & \\
\hline 2 & 2 & 1 & 1.447 & .246 & 1.91 \\
\hline 3 & 3 & 1 & 1.818 & .389 & 2.61 \\
\hline 4 & 4 & 1 & 2.139 & .675 & 3.09 \\
\hline 5 & 5 & 1 & 2.531 & .461 & 3.48 \\
\hline
\end{tabular}

*Aclapted from Brown (1931) Table 6. Brown did not include a $1: 1$ display. 
Table 2

Ratio of Velocities (Standard/Variable) for Perceived Equality of Velocity for Binocular and Monocular Viewing

\begin{tabular}{|c|c|c|c|c|c|c|}
\hline \multirow{3}{*}{$\begin{array}{l}\text { Distance (m) } \\
\text { of Standard } \\
\text { Display }\end{array}$} & \multirow{3}{*}{$\begin{array}{c}\text { Constancy } \\
\text { Ratio }\end{array}$} & \multirow{3}{*}{$\begin{array}{c}\text { Angular } \\
\text { Velocity } \\
\text { Ratio }\end{array}$} & \multicolumn{4}{|c|}{ Obtained Ratio of Velocities } \\
\hline & & & \multicolumn{2}{|c|}{ Binocular } & \multicolumn{2}{|c|}{ Monocular } \\
\hline & & & Mean & SD & Mean & SD \\
\hline 1 & 1 & 1 & .971 & .104 & .967 & .130 \\
\hline 2 & 1 & 2 & 1.025 & .079 & .944 & .140 \\
\hline 3 & 1 & 3 & 1.100 & .130 & .957 & .158 \\
\hline 4 & 1 & 4 & 1.014 & .131 & 1.104 & .303 \\
\hline 5 & 1 & 5 & 1.188 & .201 & 1.128 & .201 \\
\hline
\end{tabular}

Note-Variable display was always at a distance of $1 \mathrm{~m}$; physical sizes of standard and variable frames were equal.

\section{EXPERIMENT 3}

Experiment 3 was designed to substantiate the conclusion of Experiment 2 by providing a direct demonstration that the conditions of monocular viewing in Experiment 2 actually did produce different perceptions of relative distance from the distance perceptions under the binocular conditions. A second aim of Experiment 3 was to contribute data toward an assessment of the role of phenomenal size in the frame effects observed in Experiments 1 and 2. Rock, Hill, and Fineman (1968) have argued that the perceived extent of the movement path is a critical datum for the velocity algorithm, and Gogel (1970) has proposed that "a definition of the transposition effect at either the distal or proximal level is inadequate" and "the perception of speed is related to the $S$ ' $/ \theta$ (perceived size/visual angle) of the frames within which the movement appears and is not determined by the physical or retinal sizes of the frame per se" (p. 168).

\section{Method}

Stimuli. The stimuli were the rectangular frames displayed in the dark. No moving points were incluede. One frame, $7.5 \times$ $2.5 \mathrm{~cm}$ at a distance of $1 \mathrm{~m}$, served as the modulus. The other frames and the distances at which they were presented are listed in Table 3 in the columns headed "Variable Frame."

Subjects. Twelve undergraduates, six men and six women, served as subjects, six assigned to binocular viewing and six to monocular viewing.

Procedure. Each subject provided four judgments of the relative size and the relative distance of each of the nine size-distance combinations listed in Table 3. For half of the subjects, all of the size judgments were secured first, followed by the distance judgments; for the other subjects, the order of judgments was reversed. The 36 trials were in random order. The size and the distance of the modulus were assigned the number 10 and were present on every trial. The following is a paraphrase of the instructions: In this experiment you will estimate the size of a frame and the distance it is away from you. The display to your right is to serve as the standard. I have arbitrarily assigned the number 10 to the distance the frame is from you. This number does not represent feet, yards, or any familiar unit; it is just an arbitrary number. Your task is to assign a whole number to the size and to the distance of the frame relative to the size and distance of the standard. Use the numbers in a consistent way for size and distance. That is to say, if both the size of the opening in the frame and the distance of the frame on your left appears to be twice as large and twice the distance of the standard frame on your right, you would assign the number 20 to both the size and distance of this frame. (Other examples were added whenever the subjects indicated that the task was not understood.)

\section{Results}

The geometric mean magnitude estimates of the relative sizes and distances of the variable frames are shown in Table 3 for the two conditions of viewing. The first five rows show the estimates when the variable and modulus were both at a distance of $1 \mathrm{~m}$ from the subject. The magnitude estimates of distance show that the two frames were judged to be approximately equidistant and that the judgments were not affected much by viewing condition. The size estimates of the equidistant frames

Table 3

Geometric Mean Magnitude Estimates of the Size and Distance of Frames with Binocular and Monocular Viewing

\begin{tabular}{|c|c|c|c|c|c|}
\hline \multicolumn{2}{|c|}{ Variable Frame } & \multicolumn{2}{|c|}{ Binocular Estimate } & \multicolumn{2}{|c|}{ Monocular Estimate } \\
\hline Size & Distance & Size & Distance & Size & Distance \\
\hline $7.5 \times 2.5$ & 1 & 8.25 & 11.90 & 10.04 & 11.30 \\
\hline $15.0 \times 5.0$ & 1 & 26.18 & 11.07 & 30.69 & 8.44 \\
\hline $22.5 \times 7.5$ & 1 & 36.14 & 11.30 & 39.65 & 10.38 \\
\hline $30.0 \times 10.0$ & 1 & 59.24 & 9.73 & 49.52 & 8.33 \\
\hline $37.5 \times 12.5$ & 1 & 69.95 & 8.43 & 70.73 & 8.43 \\
\hline $15.0 \times 5.0$ & 2 & 23.76 & 30.42 & 12.13 & 19.15 \\
\hline $22.5 \times 7.5$ & 3 & 34.57 & 55.26 & 14.92 & 20.47 \\
\hline $30.0 \times 10.0$ & 4 & 49.31 & 70.56 & 14.71 & 24.55 \\
\hline $37.5 \times 12.5$ & 5 & 51.31 & 90.05 & 12.98 & 23.89 \\
\hline
\end{tabular}

Note-The modulus was alway the $7.5 \times 2.5 \mathrm{~cm}$ frame at a distance of $1 \mathrm{~m}$ and was assigned the value of 10 both for size and distanc'. 
were linearly related to the relative objective size with a marked tendency toward overestimation.

Of greater interest are the data in the first row of Table 3 coupled with the data in the last four rows. For these five rows, the physical size and distance of the frames varied while the visual angles subtended by the frames were invariant and equal to the visual angle of the modulus. Table 3 shows that, despite the fact that the two displays subtended the same angle the relative objective sizes and relative objective distances were discriminated when binocular viewing was allowed. In contrast, under conditions of monocular viewing, neither relative objective size nor distance was discriminated. For convenience of inspection, the data for distance estimation have plotted in Panel a of Figure 3 and the data for size estimation in Panel c.

\section{Conclusion}

It is plain that the conditions of viewing (binocular vs. monocular) affected the perceived relative distances and sizes of the frames significantly. Panel b of Figure 3 reproduces the velocity data of Experiment 2. It is equally clear that the same manipulation of viewing condition did not affect relative perceived velocity. Comparison of the three panels of Figure 3 bolsters the conclusion of Experiment 2, that the effect of the frame on perceived velocity is not influenced by the availability of distance cues or the perceived distance of the frame. In addition, Figure 3 implies that perceived size need not be taken into account in deriving predictions concerning perceived relative velocity. Relative retinal factors are sufficient.

\section{EXPERIMENT 4}

The hypothesis that perceived velocity depends on relative retinal displacement per unit of time is a general account of perceived velocity. Although the application to constancy has been emphasized, the hypothesis is not designed simply as an account of constancy. In the present experiment, we demonstrate large deviations from speed constancy, which, nonetheless, are strictly predictable from the hypothesis of relational determination. As in Experiment 2, the standard frames in Experiment 4 were presented at various distances. However, unlike Experiment 2, in which the physical size of the standard frame was constant, the physical size of the frame in Experiment 4 was varied in direct proportion to its distance from the observer, so that the retinal size of the frame was constant. As a consequence, in contrast to Experiment 2, in which the angular velocity and the angular size of the frame varied concomitantly as distance varied, in Experiment 4, as distance increased, the angular velocity steadily diminished while the angular size of the frame remained unchanged. Under these latter circumstances, if the two displays (variable and
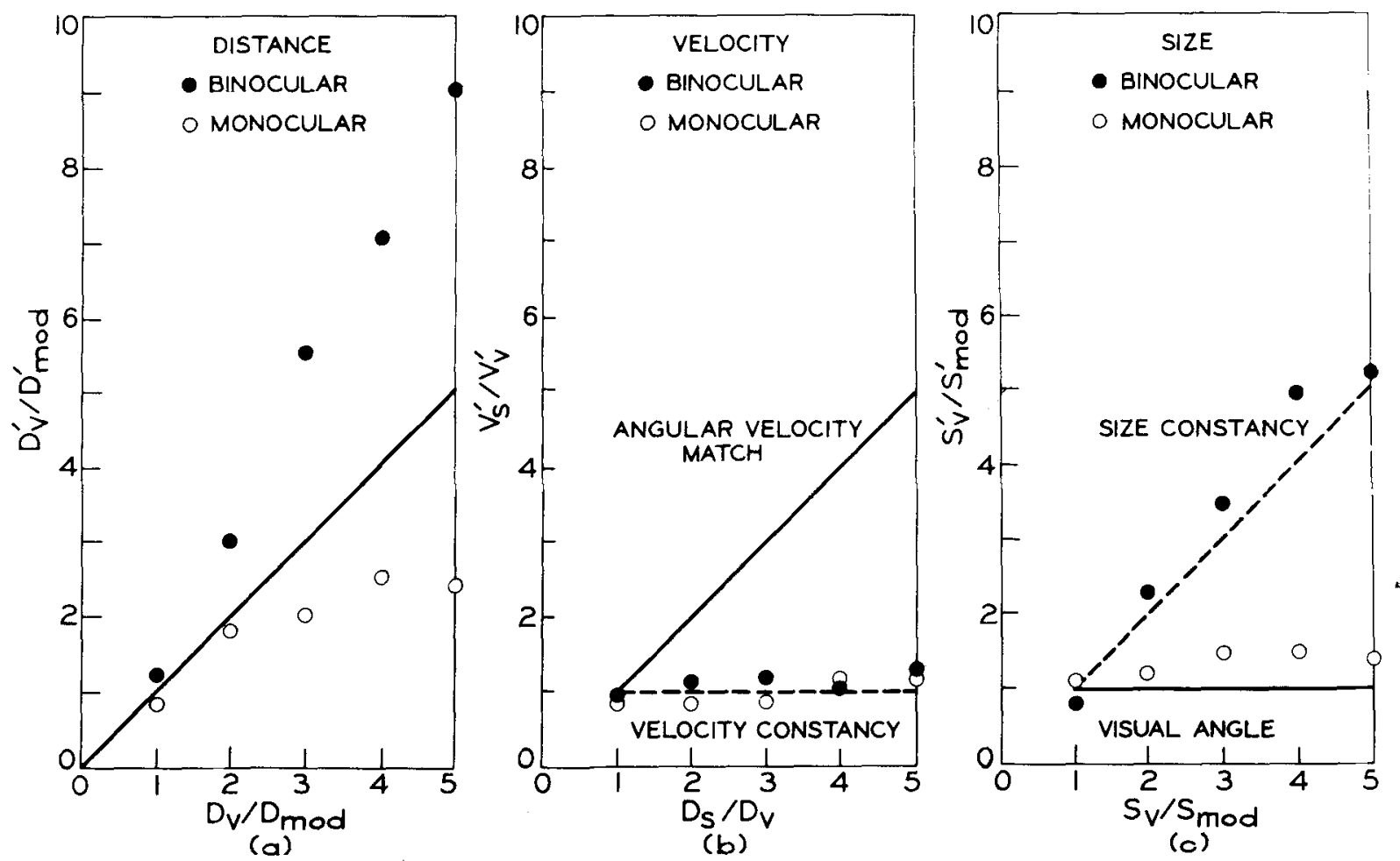

Figure 3. Panels a and $\mathrm{c}$ show the results of the magnitude estimation tasks in Experiment 3 for comparison with the results for velocity matching in Experiment 2 shown in Panel b. 
standard) appear to have the same velocity only when the moving targets in the two fields traverse the same fraction of their respective retinal fields in the same time, then the objective linear velocity in the nearby display will have to be adjusted downward with each successive increase in the distance of the variable. In fact, the relative angular velocity hypothesis predicts that the ratio of objective velocities (standard/variable) accepted as a satisfactory match by the subject should correspond to the ratio of objective distances of the two displays.

\section{Method}

Stimuli. The standard velocity displays were the five rectangular frames, $7.5 \times 2.5$ to $37.5 \times 15.0 \mathrm{~cm}$, presented for binocular viewing in dark surroundings at appropriate distances from 1 to $5 \mathrm{~m}$ from the subject. The two velocities, 2.5 and $7.5 \mathrm{~cm} / \mathrm{sec}$ used in Experiments 1 and 2, were used again. The variable velocity display was $7.5 \times 2.5 \mathrm{~cm}$ at a distance of $1 \mathrm{~m}$.

Subjects. Three men and three women undergraduate students served as subjects.

Procedure. Each subject provided $\mathbf{4 0}$ velocity judgments using the method of adjustment: 5 standard displays $\times 2$ standard velocities $\times 4$ replications in four randomized blocks of 10 trials each.

\section{Results}

Table 4 shows the mean obtained velocity ratios. When both displays were the same size and distance, the ratio did not differ from 1.0. This is simply evidence that the matching task can be performed with high accuracy when there are no perturbing factors. However, when differences in the distances of the displays were introduced, large and systematic deviations from objective equality and speed constancy resulted. The obtained ratios of velocities varied in the manner predicted by the relational hypothesis. The magnitude of the effect may be appreciated by comparing the data in Table 4 with the ratios for binocular viewing reported for Experiment 2 in Table 2.

\section{Conclusion}

The relational hypothesis can account for striking deviations from constancy as well as for constancy. We may also note that, inasmuch as the outcome of Experiment 3 implies that percieved relative size and perceived relative distance were discriminated under the conditions of Experiment 4, there is additional reason for doubting that either perceived size or distance of the frames plays a role in determining perceived velocity when frames are present.

\section{EXPERIMENT 5}

The displays examined in Experiments 1-4 have been two-element arrangements of a moving spot and surrounding frame. In such simple contexts, identifying the framework of movement does not pose a problem. However, the conditions of viewing outside the laboratory typically are not simple. The normally articulated visual scene will project a great variety of contours onto the retina. These contours, removed from the path of movements laterally and in depth by various amounts, will also be displaced retinally with respect to the path of movement by various degrees. The contours outside the immediate ones constituting the movement frameworks may be transposed in their respective fields in the same proportion as the frameworks, or the "outside" contours may be nontransposed.

Under such circumstances, which retinal contours constitute the effective framework in determining the relational effect? Consider two opposed answers to this question: (1) Weighting. All of the contours are entered into the processing of velocity, their contribution varying as a function of spatial proximity and other factors. Wallach (1939) may have had this formulation in mind when he attributed the diminution of the transposition effect in shifting from dark room to normal illumination to the introduction of nontransposed elements which become visible only in normal illumination. (2) Separation of systems. Only the contours most adjacent to the moving object enter into the process; all other contours are ignored. There is a "separation of systems" similar to that which Duncker (1929) found to be operative in determinations of induced movement.

Experiment 5 was designed to provide evidence relevant to a decision between these two formulations. In Part $\mathrm{A}$ of the experiment, velocity matches were

Table 4

Ratio of Velocities (Variable/Standard) for Perceived Equality of Velocity When Distance of Variable Frame Varied, Projective Sizes of Variable and Standard Frames Equal (Experiment 4)

\begin{tabular}{|c|c|c|c|c|c|c|}
\hline \multicolumn{2}{|c|}{ Variable Frame } & \multirow{2}{*}{$\begin{array}{l}\text { Ratio of Projective } \\
\text { Sizes of Frames } \\
\text { (Variable/Standard) }\end{array}$} & \multicolumn{2}{|c|}{$\begin{array}{c}\text { Predicted Ratio } \\
\text { of Velocities }\end{array}$} & \multicolumn{2}{|c|}{$\begin{array}{c}\text { Obtained Ratio } \\
\text { of Velocitics }\end{array}$} \\
\hline Size $(\mathrm{cm})$ & Distance $(\mathrm{m})$ & & Hypothesis & Constancy & Mean & $\mathrm{SD}$ \\
\hline $7.5 \times 2.5$ & 1 & 1 & 1 & 1 & 1.123 & .160 \\
\hline $15.5 \times 5.0$ & 2 & 1 & 2 & 1 & 1.845 & .202 \\
\hline $22.5 \times 7.5$ & 3 & 1 & 3 & 1 & 2.722 & .264 \\
\hline $30.0 \times 10.0$ & 4 & 1 & 4 & 1 & 3.604 & .185 \\
\hline $37.5 \times 15.0$ & 5 & 1 & 5 & 1 & 4.756 & .452 \\
\hline
\end{tabular}

Note-Standard dinnla : as dwats the $7.5 \times 2.5 \mathrm{~cm}$ frame at a distance of $1 \mathrm{~m}$ 
obtained with four different displays. Each display presented the $3.1(22.5 \times 7.5$ vs. $7.5 \times 2.5 \mathrm{~cm})$ rectangular frame-moving spot arrangement of the earlier experiments. Display 1 presented the frames and spots in total darkness without any other visible contours. In Display 2, the rectangular frames were flanked by pairs of 9 -mm-wide vertical lines equal in length to the vertical sides of their respective frames (see Figure 4) and in the same plane as the frames. The separation between the frame and the inside flanking line was $9 \mathrm{~mm}$, as was the separation between the two lines in a pair. In this case, the outside elements were transposed in the same proportion as the frames. Displays 3 and 4 presented outside elements which were nontransposed. As Figure 4 shows, this was accomplished by introducing pairs of equal-length flanking lines alongside the two frames.

If contours outside the spatially proximate frame are weighted into the process of relational determination of velocity, then Displays 3 and 4 which include nontransposed flanking lines should yield a smaller transposition effect of velocity than Display 1 . Display 2, which includes flanking lines that are transposed in the same proportion as the frames, should yield an enhanced effect. On the other hand, if separation of systems is complete, the "outside" elements should not influence perceived velocity and the matches should not differ among the displays.

Part $B$ was addressed to the same question. In Part B, the outside elements were complete enclosing frames. In Display $T$, each proximate frame was surrounded by a superordinate frame whose linear dimensions were $37.5 \times 12.5$ and $12.5 \times 5 \mathrm{~cm}$ so that they were transposed relative to each other in a 3:1 ratio, consistent with the proximate frames. In Display NT, the large superordinate frame surrounded both the small and the large proximate frame. Display St was the standard 3:1 arrangement without visible outside contours. Expectations in Part B were the same as in Part A.

\section{Method}

Subjects. Six subjects served in Part A and six other subjects in Part B. In each group, there were three men and three women.

Procedure. After a practice series of 8 settings, each subject provided 64 velocity matches, 16 for each of the four display types. Matches for each display type were blocked, but the four types

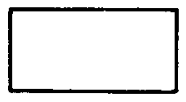

I
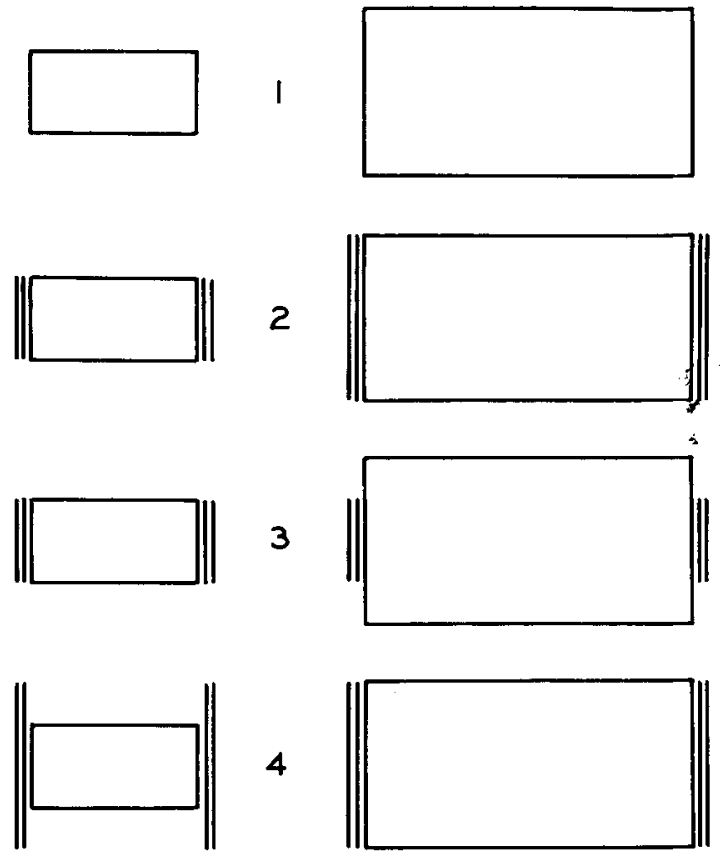

Figure 4. Displays used in Part A of Experiment 5.

were presented in random order to each subject. For each block of 16 trials, the small- and large-frame displays served as variables in regular alternation. Four standard velocities, 4, 8, 12 , and $16 \mathrm{~cm} / \mathrm{sec}$, were each presented four times in each block of 16 trials in random order, with the exception that each velocity was paired with the standard frame size exactly twice.

The procedure of Part B followed the procedure of Part A in all essential respects.

\section{Results}

Table 5 shows the mean transposition effects averaged for the small-frame variable and largeframe variable in Part $\mathbf{A}$. There were no significant differences in magnitude of the transposition effect associated with any of the factors. The overall mean transposition effect (ratio of velocities) was 2.22, with the means for individual displays ranging from 2.14 to 2.32 and the means for the four velocities ranging frome 2.13 to 2.27 .

Table 6 shows the mean transposition effects averaged for the small inner frame variable and the large inner frame variable in Part B. There were no

Table 5

Ratio of Velocities for Perceived Equality of Velocity for Four Movement Displays

\begin{tabular}{|c|c|c|c|c|c|}
\hline \multirow[b]{2}{*}{ Display } & \multicolumn{4}{|c|}{ Standard Velocity $(\mathrm{cm} / \mathrm{sec})$} & \multirow{2}{*}{$\begin{array}{l}\text { Grand } \\
\text { Mean }\end{array}$} \\
\hline & 4 & 8 & 12 & 16 & \\
\hline $\begin{array}{l}\text { 1. No Outside Elements } \\
\text { 2. Outside Elements Transposed } \\
\text { 3. Outside Elements Nontransposed } \\
\text { 4. Outside Elements Nontransposed }\end{array}$ & $\begin{array}{l}2.14 \\
2.22 \\
2.09 \\
1.98\end{array}$ & $\begin{array}{l}2.12 \\
2.27 \\
2.43 \\
2.17\end{array}$ & $\begin{array}{l}2.22 \\
2.22 \\
2.45 \\
2.16\end{array}$ & $\begin{array}{l}2.18 \\
2.28 \\
2.21 \\
2.25\end{array}$ & $\begin{array}{l}2.17 \\
2.25 \\
2.32 \\
2.14\end{array}$ \\
\hline Grand Means & 2.13 & 2.25 & 2.26 & 2.23 & \\
\hline
\end{tabular}

Note $-A$ ratio of 1.0 indicates zero transposition of velocitv; a ratio of 3.0 represents perfect transposition. 
Table 6

Ratio of Velocities for Perceived Equality of Velocity for Four Movement Displays

\begin{tabular}{lccccc} 
& \multicolumn{4}{c}{ Standard Velocity (cm/sec) } \\
\cline { 3 - 6 } Display & 4 & 8 & 12 & \multicolumn{1}{c}{$\begin{array}{c}\text { Grand } \\
\text { Means }\end{array}$} \\
\hline St. No Superordinate Frames & 2.43 & 2.56 & 2.49 & 2.41 & 2.47 \\
T. Transposed Superordinate Frames & 2.65 & 2.82 & 2.31 & 2.36 & 2.57 \\
NT. Nontransposed Superordinate Frames & 2.65 & 2.38 & 2.39 & 2.30 & 2.42 \\
Grand Means & 2.58 & 2.59 & 2.39 & 2.36 \\
\hline
\end{tabular}

Note-A ratio of 1.0 indicates zero transposition of velocity; a ratio of 3.0 represents perfect transposition.

significant differences in magnitude of the transposition effect associated with any of the factors. The overall mean transposition effect was 2.48 , with the means for the three displays ranging from 2.42 to 2.54 and the means for the four velocities ranging from 2.36 to 2.59 .

\section{EXPERIMENT 6}

Experiment 5 suggests that a complete separation of systems occurs in the determination of perceived relative velocity. However, inasmuch as all the elements in each display were coplanar, it may be questioned whether similar results would be obtained for "outside" contours distributed in depth. Admittedly, on a priori as well as empirical grounds [see Gogel's (1970) discussion of the adjacency principle], the coplanar arrangement may seem to be the most probable to yield an effect of outside elements. Nevertheless, a typical setting affords exposure to contours distributed in depth and, in the interests of ecological validity, these conditions should be examined.

Experiment 6 was designed to compare velocity matches secured for two retinally equivalent pairs of 3:1 displays, one pair correlated with two frames of different size at the same distance and the other pair correlated with equisized frames at 3:1 distance ratios. These two types of display pairs were presented either in total darkness or in normal illimination. Darkroom conditions afforded no view of outside contours; normal illumination afforded exposure to a diverse array of outside contours in the same plane as the displays and also separated in depth from the displays.

The experiment was addressed to three questions: (1) Will separation of systems occur for the multiple contour condition, e.g., will transposition of velocity be reduced in normal illumination? Brown (1931) found this to be the case. (2) Will speed constancy be enhanced by normal illumination? (3) Will individual differences in the transposition effect be correlated with differences in speed constancy?

\section{Method}

Stimuli. The frames were two $22.5 \times 7.5 \mathrm{~cm}$ rectangles and one $7.5 \times 2.5 \mathrm{~cm}$ rectangle. For transposition tests, one large and one small frame were presented at a distance of $1 \mathrm{~m}$, as in Experiment 5. For speed constancy tests, the two large frames were used, one at a distance of $1 \mathrm{~m}$ and the other at a distance of $3 \mathrm{~m}$ from the viewing position. The four standard velocities of Experiment 5 were used again. Dark room conditions provided views of only the frames. Normal illumination was supplied by a fluorescent table lamp positioned behind the subject, providing a view of a scene with the normal complement of surfaces and edges associated with tables, cabinets, apparatus, etc.

Subjects. The subjects were six men and six women undergraduate volunteers.

Procedure. Following eight practice trials, each subject provided 16 velocity judgments under each of the four conditions. For six subjects, the darkroom conditions were tested first; for the other subjects, the illuminated conditions were tested first. Standard velocity and standard frame were counterbalanced, as in Experiment 5A.

\section{Results}

Analysis of variance revealed a sizable main effect of Test Type $[F(1,11)=136.22]$ and a significant Test Type by Illumination Conditions interaction $[\mathrm{F}(1,11)=5.74, \mathrm{p}<.05]$. There were no other significant effects. The main effect of Test Type is familiar; the velocity ratios approximate 1.0 (speed constancy) when equisized frames are presented at different distances but show a substantial transposition effect when equidistant frames of different size are presented. Table 7 shows the mean velocity ratios involved in the Test Type

Table 7

Mean Ratio of Velocities for Perceived Equality of Velocity for Two Retinally Equivalent Display Pairs Under Two lllumination Conditions

\begin{tabular}{|c|c|c|c|}
\hline \multirow[b]{2}{*}{ Test Type } & \multicolumn{2}{|c|}{ Viewing Condition } & \multirow[b]{2}{*}{$\begin{array}{l}\text { Grand } \\
\text { Means }\end{array}$} \\
\hline & $\begin{array}{c}\text { Normal } \\
\text { Illumination }\end{array}$ & Dark & \\
\hline $\begin{array}{l}\text { Transposition Test (Equidistant, 3:1 Size) } \\
\text { Speed Constancy Test (Fquisized, 3:1 Distance) }\end{array}$ & $\begin{array}{r}2.09 \\
.92\end{array}$ & $\begin{array}{r}2.18 \\
.85\end{array}$ & $\begin{array}{r}2.13 \\
.88\end{array}$ \\
\hline Grand Means & 1.50 & 1.51 & \\
\hline
\end{tabular}


by Illumination Conditions interaction. Viewing the displays in normal lighting reduced the transposition effect in the equal distances condition but enhanced speed constancy in the equal frames condition. These effects were small relative to the overall means of transposition and speed constancy (rightmost column). Inspection of individual subjects' data showed a reduced transposition effect in 5 of 12 subjects, enhanced speed constancy in 8 of 12 subjects, and both effects in only 3 of 12 subjects.

For both illumination conditions, a Pearson correlation was computed for velocity ratios obtained from the equal distances and equal frames conditions. Individual scores of transpostion effect were unrelated to speed constancy ratios regardless of whether the displays were viewed under normal illumination $(r=.35)$ or in darkness $(r=.17)$.

\section{Conclusion}

The results of Experiment 5 provided no evidence that contours in the plane of movement other than the most proximal ones affect perceived velocity. Experiment 6 uncovered small differences arising from mainpulation of level of illumination which it seems reasonable to attribute to the introduction of extraneous contours in the lighted room condition. However, the individual subjects' data show that most subjects did not conform unequivocally to the trend. ${ }^{2}$

\section{DISCUSSION}

The present results confirm Brown's conclusion that relative object-relative displacement contributes significantly to the determination of perceive relative velocity. The results also justify Wallach's claim that Brown's transposition principle applied to retinal patterns can account for speed constancy.

Nevertheless, the results do not show that the relational hypothesis is sufficient to explain perceived speed at a distance under all circumstances. Other investigators have reported and we have confirmed in our laboratory that the perceived velocity of a single point of light observed in uniformly dark surroundings will exhibit constancy when distance from the observer varies. The relational hypothesis cannot be applied to these conditions, since no other objects or frameworks are visible. Another mechanism must be operative in this case.

Adopt an alternative mode of seeing Figure 1 and take it to be a surrogate for the street scene outside the window. Considered in this way, a different account of percieved velocity at a distance is suggested. This explanation attributes invariance of perceived velocity to appropriate registration of distance. Two versions of this explanation may be distinguished:
(1) Extent mediation. Appropriate registration of distance allows an extent (size) constancy mechanism to operate. This mechanism insures that the perceived extent of the path of movement corresponds to its true extent despite the diminishing projection of the movement path. Assuming that perceived velocity $\left(\mathrm{V}^{\prime}\right)$ is a function of perceived space $\left(\mathrm{S}^{\prime}\right)$ per unit of time $\left(T^{\prime}\right)$, if $S^{\prime}$ (far) $=S^{\prime}$ (near) then $V^{\prime}$ far will appear to equal $V^{\prime}$ near when $T^{\prime}$ (far) = $T^{\prime}$ (near). Constancy will prevail. An experiment by Rock, Hill, and Fineman (1968) has been, interpreted as support for the extent mediation hypothesis. Rock et al. demonstrated that reducing the availability of distance cues caused velocity judgments to tend toward angular velocity matches and size judgments to tend toward visual angle matches. Rock et al. concluded that speed constancy depends on size constancy.

(2) Distance calibration. The alternative version proposes that absolute angular velocity (A) is calibrated by registering distance $\left(D^{\prime}\right)$ according to a velocity algorithm, $\mathbf{V}^{\prime}=\mathbf{A} \times \mathrm{D}^{\prime}$, analogous in form to the size-distance invariance hypothesis. The value of $\mathbf{V}^{\prime}$ will remain invariant as long as $A \times D^{\prime}$ remains constant, and this state of affairs will prevail whenever $D^{\prime}($ far $) / D^{\prime}$ (near) $=D($ far $) / D$ (near) A series of experiments by Wist, Diener, and Dichgans (1976) and Wist, Diener, Dichgans, and Brandt (1975) bears on the distance-calibration hypothesis. These experiments have manipulated the perceived distance of a movement field by varying the oculomotor cues or binocular disparity and have measured perceived velocity for targets moving at the same angular velocity. Perceived velocity was found to vary directly with perceived distance, e.g., if the convergence-equivalent distance was increased, perceived velocity increased.

The visual system appears to be capable of exploiting two different types of information for the determination of object velocity. This capability is useful inasmuch as the two factors (object-relative displacement and distance calibration) can provide different kinds of information about velocity.

Information about absolute (egocentric) velocity depends on the distance calibration operation, in one of the two forms hypothesized above. That is, resolution of the question How fast is the object moving across the field? cannot be accomplished by relying exclusively on object-relative displacement. The egocentric distance of the object must be taken into account, In the absence of distance cues, an observer viewing a single moving object traversing a field of unfamiliar dimensions would not know the objective linear velocity of the object. The fact that the object is undergoing displacement relative to the field would convey no information about its objective rate of displacement. For discrimination of 
objective velocity, either the algorithm $\mathrm{V}^{\prime}=\mathrm{A} \times \mathrm{D}^{\prime}$ or the algorithm $V^{\prime}=S^{\prime} / T^{\prime}$ must be applied. In both cases, distance must be registered and processed. Since the distance calibration operation can provide scalar information, relative velocity information can also be derived by comparing the separately determined absolute velocities of the objects for comparison.

The utility of relative angular velocity (relative object-relative displacement) is limited to discrimination of relative object velocity, that is, to resolving the question: Is $A$ moving faster or slower than $B$ and in what proportion? In fact, for this purpose, relative angular velocity is the factor par excellence providing more direct access to relative object velocity than the distance calibration mechanism, since application of the latter mechanism requires additional operations. However, relative angular velocity is uninformative about absolute object velocity. The correctness of this assertion is suggested by considering how the outcome of Experiment 2 would have been affected if the subject had been incapable of registering the absolute velocity of the standard. It seems likely that the results for this imaginary subject would not have differed from the results secured with our normal subjects who could discriminate absolute velocity. The imaginary subject, like the real subject, equating rates of object-relative displacement, would exhibit constancy despite the fact that the standard would have a reliable perceived absolute velocity only for the real subject. ${ }^{3}$

\section{REFERENCES}

Brown, J. F. The visual perception of velocity. Psychologische Forschung, 1931, 14, 199-232.

Conen, R. L. Problems in motion perception. Uppsala: Appelbergs, 1964.

DuNCKER, K. Über induzierte Bewegung. Psychologische Forschung, 1929, 12, 180-259.

Gibson, J. J. The perception of the visual world. New York: Houghton Mifflin, 1950.

GiBson. J. J. The senses considered as perceptual systems. New York: Houghton Mifflin, 1966.

Gogel, W. C. The adjacency principle and three-dimensional illusions. Psychonomic Monograph Supplement, 1970, 3 (Whole No. 45).

Gogel, W. C. The metric of visual space. In W. Epstein (Ed.), Stability and constancy in visual perception: Mechanisms and processes. New York: Wiley-Interscience, 1977.
Hochberc, J. E. Perception (2nd ed.). Englewood Cliffs. N.J: Prentice-Hall. 1978.

Rock. I. An introduction to perception. New York: Macmillan. 1975.

Rock, I., Hil. , A. \& Fineman, M. Speed constancy as a function of size constancy. Perception \& Psychophysics, 1968, 4, 37.40.

W Allach, H. On constancy of visual speed. Psychological Review, 1939, 46, 541.442.

Wallach, H. Perception of motion. Scientific American, 1959. 201, 56-60.

Wist, E. R., Diener, H. C., \& Dichgans, J. Motion constancy dependent upon perceived distance and the spatial frequency of the stimulus pattern. Perception \& Psychophysics. $1976,19,485-491$.

Wist, E. R., Diener, H. C., Dichgans, J., \& Brandt, T. Perceived distance and the perceived speed of self-motion: Linear vs. angular velocity. Perception \& Psychophysics, 1975. 17. $549-554$

\section{NOTES}

1. Cohen (1964, Experiment 19) conducted a replication of Brown's basic experiment and also obtained an effect that was much smaller than the effect reported by Brown. Cohen tested only the $2: 1$ frame size relationship and found a velocity transposition effect of 1.25 .

2. Contrary to the results of Experiments 5 and 6, Brown (1931) reported that reducing the homogeneity of the surrounding field resulted in a significant diminution of the transposition effect. In normal illumination with two fields having a $4: 1$ ratio of linear dimensions, the velocity ratio was 2.8 when the backgrounds were a uniform black, but only 1.92 when the posterboard surrounding the rectangular windows was covered by a wallpaper pattern composed of small squares. We have no data to bring to bear on a resolution of the discrepancy between Brown's results and our failure to adduce evidence that elements other than the immediately proximate contours affect perceived velocity. It may be that Brown's display which provided exposure to a regular succession of landmarks along the edges of the window may have encouraged the subject to adopt matching strategems that overrode relative object-relative displacement. Unlike our displays which presented articulated proximate frameworks and segregated "outside" elements, Brown's displays consisted of points moving through windows cut in patterned posterboard. The absence of prominent contours separating the interior from the exterior portions of the display could have contributed to the tendency to use elements of the pattern at the edges as landmarks.

3. The need to distinguish between absolute and relative cues has been discussed in detail by Gogel (1977), who has also proposed a theory of visual space perception which emphasizes the interaction of absolute and relative sources of information.

(Received for publication April 28, 1978; revision accepted June 26, 1978.) 